\title{
Nanosecond magnetic relaxation processes in ultrathin metallic films prepared by MBE
}

\author{
R. Urban, B. Heinrich, G. Woltersdorf, K. Ajdari, K. Myrtle, and J. F. Cochran \\ Simon Fraser University, 8888 University Drive, Burnaby, BC, Canada V5A 1S6 \\ E. Rozenberg \\ Physics Department, Ben Gurion University, Beer-Sheva, POB 653 84105, Israel
}

(Received 27 August 2001; published 3 December 2001)

\begin{abstract}
Magnetic relaxation processes were investigated by ferromagnetic resonance (FMR) using crystalline $\mathrm{Cr} /$ $\mathrm{Fe} / \mathrm{GaAs}(001)$ ultrathin film structures grown by molecular beam epitaxy (MBE). For Fe films thicker than 1.5 $\mathrm{nm}$ the extrinsic relaxation term showed evidence of two magnon scattering. Above $10 \mathrm{GHz}$ the in-plane FMR linewidth was linearly dependent on the microwave frequency with an appreciable zero-frequency offset. The zero frequency offset measures the strength of extrinsic damping. The in-plane Gilbert damping includes both the intrinsic and extrinsic Gilbert contributions in qualitative agreement with recent predictions by Arias and Mills.
\end{abstract}

DOI: 10.1103/PhysRevB.65.020402

PACS number(s): 76.50.+g, 75.70.-i, 75.30. $-\mathrm{m}$

\section{INTRODUCTION}

The small lateral dimensions of spintronics devices and high-density memory bits require the employment of magnetic ultrathin film structures where the magnetic moments across the film are locked together by exchange coupling. The ultrathin films can be considered as giant magnetic molecules which have unique magnetic properties of their own. ${ }^{1}$ Spintronics and high-density magnetic recording employ fast magnetization reversal processes. It is currently of considerable interest to acquire a thorough understanding of the spin dynamics and magnetic relaxation processes in the nanosecond time regime.

The spin dynamics in the classical limit is given by the Gilbert equation of motion

$$
\frac{1}{\gamma} \frac{\partial \mathbf{M}}{\partial t}=-\left[\mathbf{M} \times \mathbf{H}_{\mathrm{eff}}\right]+\frac{G}{\gamma^{2} M_{s}^{2}}\left(\mathbf{M} \times \frac{\partial \mathbf{M}}{\partial t}\right)
$$

where $\mathbf{M}$ is the magnetization vector, $M_{s}$ is the saturation magnetization, and $\gamma$ is the absolute value of the electron gyromagnetic ratio. The effect field $\mathbf{H}_{\mathrm{eff}}=-\partial U_{\mathrm{mag}} / \partial \mathbf{M}$, where $U_{\mathrm{mag}}$ is the Gibbs free energy of the system. The first term on the right-hand side represents the precessional torque and the second term represents the relaxation term which is expressed by the well known Gilbert damping torque. ${ }^{1}$ The Gilbert damping coefficient $G$ in metals arises from the spin orbit interaction of itinerant electrons. ${ }^{2}$ However, in realistic samples structural inhomogeneities and defects can play a major role in magnetic relaxation. ${ }^{3}$ The two magnon scattering process has been used extensively to describe extrinsic damping in ferrites. ${ }^{3-7}$ The Patton group pioneered the use of two magnon scattering in metallic films. ${ }^{8}$ In a wide range of metallic amorphous ribbons, ${ }^{9}$ films,${ }^{10}$ and metallic ultrathin film multilayers ${ }^{1,11,12}$ the ferromagnetic resonance (FMR) linewidth, $\Delta H$, above $10 \mathrm{GHz}$ is not only proportional to the microwave angular frequency, $\omega$, as expected from the Gilbert damping but possesses a zerofrequency offset $\Delta H(0)$,

$$
\Delta H(\omega)=\Delta H(0)+1.16 \frac{\omega}{\gamma} \frac{G_{\text {eff }}}{\gamma M_{s}} .
$$

$\Delta H(0)$ is caused by magnetic inhomogeneities and therefore its origin is extrinsic. The Gilbert parameter $G_{\text {eff }}$ was used to express the fact that the slope of the FMR linewidth in Eq. (2) can in general include both intrinsic, $G_{\text {int }}$, and extrinsic, $G_{\text {ext }}$, contributions to the effective Gilbert damping parameter. It is a reasonable assumption that for samples where the zero frequency offset is negligible $[\Delta H(0)=0]$ the effective Gilbert damping $G_{\text {eff }}$ equals to the intrinsic Gilbert parameter $G_{\text {int }}$.

In amorphous ribbons it was shown ${ }^{13}$ that $\Delta H(0)$ can be explained by a two magnon scattering process where the three-dimensional (3D) Fourier transform, $F(q)$, of the sample inhomogeneities can be represented by a step function in the $q$-wave vector with the upper cutoff wave vector less than the maximum wave vector allowed by the spinwave manifold (spin-wave dispersion relation). In the above model the two magnon scattering did not affect the intrinsic Gilbert damping $G_{\text {int }}$.

In ultrathin film structures the spin-wave $k$-wave vectors are held within the film plane, ${ }^{1}$ constraining the two magnon scattering to a 2D spin-wave manifold. McMichael et al. ${ }^{14}$ found that the FMR linewidth at $9.5 \mathrm{GHz}$ for a $10 \mathrm{~nm}$ thick $\mathrm{Ni}_{80} \mathrm{Fe}_{20}$ film (PY) grown on $\mathrm{NiO}$ is affected by two magnon scattering. The 2D spin manifold in PY leads to an abrupt disappearance of the two magnon scattering contribution when the magnetization angle is inclined more than $45^{\circ}$ away from the film plane. The measured strength of the two magnon scattering was found to decrease gradually from its maximum value in the parallel configuration ${ }^{20}$ to nearly zero for the magnetic moment oriented $60^{\circ}$ away from the sample plane. The observed gradual decrease in the strength of the two magnon scattering with an increasing magnetization angle was explained by the intrinsic lifetime of magnons. It replaces the energy delta function in the two magnon process by a Lorentzian.

Recently Arias and Mills ${ }^{15}$ addressed theoretically the role of two magnon scattering in ultrathin film structures in par- 
allel configuration. They showed that lateral variations in the perpendicular uniaxial interface anisotropy field (due to the interface roughness) are a leading source of the two magnon scattering in ultrathin films. Azevedo et al. ${ }^{16}$ found in $\mathrm{Ni}_{50} \mathrm{Fe}_{50}$ sputtered films that the measured variations of the FMR linewidth and field were consistent with the two magnon scattering model proposed by Arias and Mills.

It is important to realize that for both the $2 \mathrm{D}$ and $3 \mathrm{D}$ spin-wave manifold there are no degenerate magnons with the FMR mode in the perpendicular configuration, therefore in the presence of two magnon scattering the FMR linewidth, $\Delta H_{\perp}$, should be smaller than that in the parallel configuration, $\Delta H_{\|}$. Recently we obtained samples satisfying this condition $\left(\Delta H_{\perp}<\Delta H_{\|}\right)$by preparing crystalline $\mathrm{Cr} / \mathrm{Fe}(001)$ ultrathin film structures deposited directly onto $\mathrm{GaAs}(001)$ substrates. These samples allowed us to interpret the FMR linewidths in terms of intrinsic damping and the two magnon scattering mechanism.

The purpose of this paper is to study the intrinsic and extrinsic relaxation mechanism in $\mathrm{Cr} / \mathrm{Fe} / \mathrm{GaAs}(001)$ ultrathin film structures as a function of the microwave frequency and the angle $\theta$ between the external magnetic field $\mathbf{H}$ and the sample plane, and to compare the extrinsic damping with the Arias and Mills model calculations. ${ }^{15}$

\section{RESULTS AND DISCUSSION}

Magnetic $\mathrm{Cr} / \mathrm{Fe}(001)$ structures were deposited by molecular beam epitaxy (MBE) directly onto semi-insulating $\operatorname{GaAs}(001)$ wafers. The GaAs(001) substrates were prepared by annealing and sputtering cycles monitored by means of reflection high energy electron diffraction (RHEED) until a well-ordered $(4 \times 6)$ reconstruction appeared. ${ }^{17,18}$

Iron films $[5,10,15,20$ monolayers (ML) $]$ were deposited at room temperature (RT) from a thermal source at a base pressure below $2 \times 10^{-10}$ Torr with a deposition rate of $\sim 1 \mathrm{ML} / \mathrm{min}$. The thickness was monitored by a crystal microbalance and RHEED intensity oscillations. The Fe films were covered by a crystalline chromium cap layer with a deposition rate of $\sim 1 \mathrm{ML} / \mathrm{min}$ at RT.

The in-plane FMR measurements were performed at 9.5, 24, and $36 \mathrm{GHz}$. The out of plane FMR measurements were performed only at 9.5 and $24 \mathrm{GHz}$ because we were not able to reach the FMR field at $36 \mathrm{GHz}$ in the perpendicular configuration. The Fe film in $\mathrm{Cr} / \mathrm{Fe} / \mathrm{GaAs}(001)$ ultrathin structures behaves like a giant magnetic molecule. The magnetic anisotropies are well described by a linear dependence on $1 / d$, where $d$ is the thickness of the Fe layer. ${ }^{1}$ The constant term and the slope of the linear dependence correspond to the bulk and interface anisotropies, respectively. The $\mathrm{Cr} / \mathrm{Fe} /$ $\mathrm{GaAs}(001)$ structures have the in-plane fourfold and uniaxial magnetic anisotropies, $K_{1}$ and $K_{u}$, and the effective demagnetizing field perpendicular to the film surface, $4 \pi M_{\text {eff }}$ $=4 \pi M_{s}-2 K_{\perp}^{S} / M_{s} d$, close to those found in bulk, modified only by well-defined interface anisotropies. $K_{\perp}^{s}$ is the perpendicular uniaxial interface anisotropy. The $\mathrm{Fe} / \mathrm{GaAs}(001)$ interface creates a uniaxial in-plane anisotropy with the hard axis along the $[1 \overline{1} 0]$ direction. ${ }^{18}$ The bulk and interface magnetic properties are as follow: $K_{1, \|}^{\text {bulk }}=4.7 \times 10^{5}$
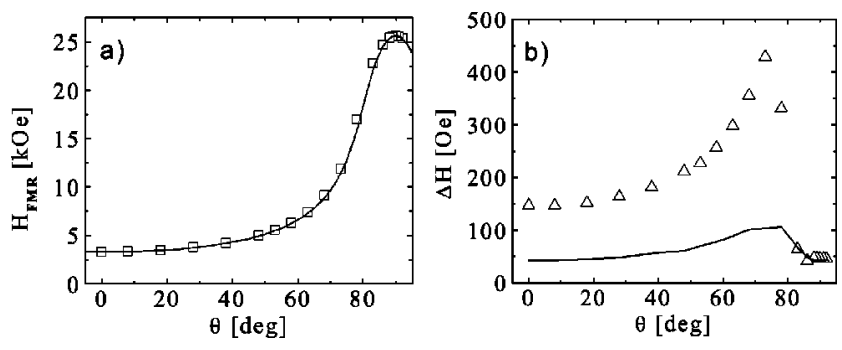

FIG. 1. (a) FMR field ( $\square$ ) and (b) FMR linewidth $(\triangle)$ at $f$ $=24 \mathrm{GHz}$ as a function of the angle $\theta$ for $20 \mathrm{Cr} / 15 \mathrm{Fe} / \mathrm{GaAs}(001)$ where the integers represent the number of ML. In-plane magnetization corresponds to $\theta=0^{\circ}$ and $\theta=90^{\circ}$ corresponds to the magnetization parallel to the sample normal. The solid line in (a) represents the fit using the magnetic parameters $4 \pi M_{\mathrm{eff}}=16.4 \mathrm{kG}$, $K_{1, \|}=3 \times 10^{5} \mathrm{ergs} / \mathrm{cm}^{3}, \quad K_{u, \|}=-3.5 \times 10^{5} \mathrm{ergs} / \mathrm{cm}^{3}, \quad K_{1, \perp}=-1.0$ $\times 10^{5} \mathrm{ergs} / \mathrm{cm}^{3}, g=2.09$. The solid line in (b) shows the calculated dependence of the FMR linewidth as a function of the angle $\theta$ using the above magnetic parameters with the Gilbert damping equal to its intrinsic value $G_{\text {int }}=1.5 \times 10^{8} \mathrm{~s}^{-1}$, see Table I.

$\operatorname{ergs} / \mathrm{cm}^{3}, \quad 4 \pi M_{s}=19.6 \mathrm{kG}, \quad K_{u, \|}^{s}=-0.09 \mathrm{ergs} / \mathrm{cm}^{2}, \quad K_{1, \|}^{s}=$ $-0.04 \mathrm{ergs} / \mathrm{cm}^{2}, K_{\perp}^{s}=0.6 \mathrm{ergs} / \mathrm{cm}^{2}$, and the spectroscopic splitting factor $g=2.09$. The superscript $s$ stands for the interface part of the magnetic anisotropies.

The FMR field and linewidth for the $20 \mathrm{Cr} / 15 \mathrm{Fe} /$ GaAs(001) sample (where the integers represent the number of ML) as a function of the angle $\theta$ are shown in Fig. 1. $\Delta H$ increases with an increasing angle, reaches a maximum around $72^{\circ}$ away from the sample plane, and then abruptly decreases to $\Delta H_{\perp}=45 \mathrm{Oe}$. The FMR linewidth $\Delta H_{\perp}$ $=45 \mathrm{Oe}$ is substantially lower than $\Delta H_{\|}=150 \mathrm{Oe}$. Clearly, two magnon scattering can contribute to the FMR linewidth. The perpendicular FMR measurements at 9.5 and $24 \mathrm{GHz}$, see Fig. 2, showed no measurable $\Delta H(0)$, and the measured slope led to the intrinsic Gilbert damping parameter $G_{\text {int }}$ $=1.5 \times 10^{8} \mathrm{~s}^{-1}$. The appreciable line narrowing shown in Fig. 1(b), and the absence of defect scattering in the perpendicular configuration, see Fig. 2, provide strong

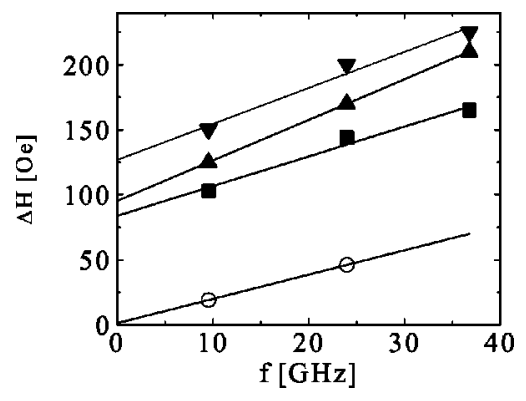

FIG. 2. The FMR linewidth for the parallel configuration for $20 \mathrm{Cr} / 15 \mathrm{Fe} / \mathrm{GaAs}(001)$ as a function of microwave frequency, $f$, measured along: $(\boldsymbol{\nabla})$ cubic axes $\{100\},(\boldsymbol{\Delta})$ hard uniaxial axis [1 $1 \overline{0} 0$, and ( $\mathbf{\square})$ direction corresponding to the easy uniaxial plane [110]. (○) represent the perpendicular FMR linewidth $\Delta H_{\perp}$ as a function of $f$. Solid lines are linear fits using Eq. (2). Note that $\Delta H(0)=0$ for the perpendicular FMR measurements. The slope of the corresponding solid line determines the intrinsic Gilbert damping $G_{\text {int }}$, see Eq. (2). 
TABLE I. The $\Delta H(0)$ and effective Gilbert damping $G_{\text {eff }}$ for the three principal in-plane and perpendicular orientations of the $20 \mathrm{Cr} / 15 \mathrm{Fe} / \mathrm{GaAs}(100)$ structure (the integers represent the number of ML). The error bars represent uncertainties in the linear fits. The accuracy of the measured FMR linewidth is a few Oe. Gilbert damping, $1.51 \times 10^{8} \mathrm{~s}^{-1}$, for the perpendicular direction [001] is the intrinsic Gilbert damping.

\begin{tabular}{lcc}
\hline \hline Direction & $\Delta H(0)(\mathrm{Oe})$ & $G_{\mathrm{eff}}\left(10^{8} \mathrm{~s}^{-1}\right)$ \\
\hline Hard uniax. axis [1]0] & $95 \pm 1$ & $2.46 \pm 0.01$ \\
Easy uniax. plane [110] & $84 \pm 9$ & $1.81 \pm 0.26$ \\
Cubic axes \{100\} & $127 \pm 11$ & $2.26 \pm 0.34$ \\
Perpendicular [001] & 0 & $1.51 \pm 0.02$ \\
\hline \hline
\end{tabular}

evidence for the presence of two magnon scattering in the $20 \mathrm{Cr} / 15 \mathrm{Fe} / \mathrm{GaAs}(001)$ structure. The $\Delta H(0)$ and Gilbert damping $G_{\text {eff }}$ for the principal in-plane and perpendicular orientations are summarized in Table I. Note that the in-plane effective Gilbert damping parameters are always bigger than the intrinsic Gilbert damping $G_{\text {int }}$.

Our comparison with Arias-Mills's theory of two magnon scattering was carried out by inserting the measured $4 \pi M_{\text {eff }}=16.4 \mathrm{kG}$ into Eq. (94) in Ref. 15. In order to produce a reasonable fit to our measured data it was required to use a large in-plane geometrical aspect ratio of the surface defects $\langle a / c\rangle=0.05$. By replacing the calculated points in the frequency range of our measurements by a linear function, see Eq. (2), we were able to evaluate corresponding extrinsic Gilbert parameter $G_{\text {ext }}$ from Arias-Mills's theory. It was found to be $2.5 \times 10^{8} \mathrm{~s}^{-1}$. The difference between the inplane effective Gilbert damping, $G_{\text {eff }}$ and the measured intrinsic Gilbert damping, $G_{\text {int }}$, does not exceed $1 \times 10^{8} \mathrm{~s}^{-1}$, see Table I. Arias-Mills's theory somewhat overestimates the extrinsic Gilbert damping, $G_{\text {ext }}$. The measured $G_{\text {ext }}\left(=G_{\text {eff }}\right.$ $\left.-G_{\text {int }}\right)$ is not small when compared to the intrinsic Gilbert damping $G_{\text {int }}$. In this respect Arias and Mills correctly pointed out that the slope which is obtained by using Eq. (2) does not necessarily represent the true intrinsic properties. The slope in $\Delta H(\omega)$ for the parallel configuration includes both the intrinsic and extrinsic contributions.

Figure 1(b) shows (the solid line) the calculated dependence of the FMR linewidth as a function of the angle $\theta$ taking for the Gilbert damping its intrinsic value $G_{\text {int }}$. The calculated increase for the intermediate angles is caused by dragging the magnetic moment behind the external applied field $\mathbf{H}$. The difference in FMR linewidth between the experimental points and the solid line in Fig. 1(b) represents the contribution of the two magnon scattering to the FMR linewidth, $\Delta H_{\text {ext }}$. The strength of two magnon scattering as a function of the angle, $\theta$, is better represented by the frequency linewidth, $\Delta \omega(\theta),{ }^{19}$

$$
\Delta \omega(\theta)=\left(\frac{\partial \omega}{\partial H}\right)_{\text {at FMR }} \Delta H_{\mathrm{ext}},
$$

where $\partial \omega / \partial H$ is evaluated for a given angle $\theta$ using the FMR condition which includes all in-plane and out-of-plane magnetic anisotropies.

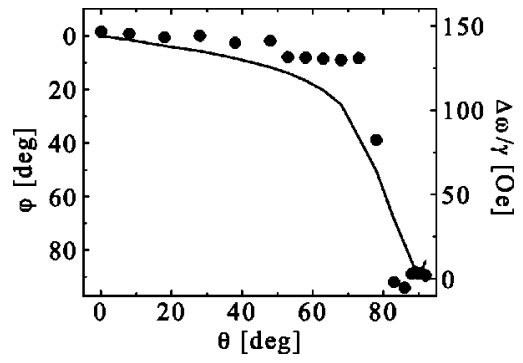

FIG. 3. (-) represent the frequency linewidth $\Delta \omega / \gamma$ [see Eq. (3)] of the two magnon scattering as a function of the angle $\theta$. The solid line represents the angle $\varphi$ between the direction of the magnetization and the sample plane as a function of the angle $\theta$, where $\theta$ is the angle between the applied field and the sample plane. The external field was rotated between the [110] in-plane and [001] perpendicular crystallographic directions.

The measured $\Delta \omega(\theta)$ is shown in Fig. 3. Remarkably, the strength of $\Delta \omega(\theta)$ stays nearly independent of $\theta$. It decreases abruptly when the magnetic moment is pulled by the field $\mathbf{H}$ towards the sample normal. This is in good agreement with the two magnon scattering predictions. The number of degenerate modes in the 2D spin-wave manifold strongly depends on the angle $\varphi$ between the saturation magnetization and the sample plane. For an angle $\theta=75^{\circ}$ the corresponding angle $\varphi=40^{\circ}$. For $\varphi=40^{\circ}$ the number of degenerate modes in our case decreases by half compared to that in the parallel configuration. For $\theta=80^{\circ}$ the number of degenerate modes is nearly zero.

A similar behavior was observed for the $20 \mathrm{ML}$ thick Fe film. The samples with 5 and $10 \mathrm{ML}$ thick Fe layers were effected by variations in the film thickness across the sample due to an enhanced role of the perpendicular uniaxial field which scales like $1 / d$. Note that these lateral variations are comparable to the sample dimensions, they are not a part of two magnon scattering mechanism. In this case the FMR linewidth is given by a simple superposition of local FMR peaks. This resulted in an additional broadening of the observed FMR peak which is particularly pronounced for the perpendicular configuration. Therefore, the perpendicular linewidth, $\Delta H_{\perp}$, did not decrease as much for the $10 \mathrm{ML}$ sample and even led to an increase for the $5 \mathrm{ML}$ sample when compared to the in-plane FMR linewidth, $\Delta H_{\|}$.

\section{SUMMARY}

FMR measurements were carried out on $\mathrm{Cr} / \mathrm{Fe} / \mathrm{GaAs}(001)$ crystalline structures at 9.5, 24, and $36 \mathrm{GHz}$. The in-plane FMR linewidth was linearly dependent on the microwave frequency. The linear slope describes an effective Gilbert damping, and the zero frequency offset comes from the extrinsic contribution to the FMR linewidth. The intrinsic Gilbert parameter was obtained with the magnetic moment oriented perpendicular to the film surface. The in-plane Gilbert damping overestimates the intrinsic damping; it includes both the intrinsic and extrinsic contribution. The extrinsic Gilbert damping can be comparable to the intrinsic Gilbert damping. This is in qualitative agreement with the recent 
calculations by Arias and Mills. ${ }^{15}$ The strength of the two magnon scattering follows the expected dependence on the angle between the external field and the sample plane. It decreases to zero when the external field pulls the magnetic moment close to the sample normal. Therefore we identify the extrinsic contribution to the FMR linewidth as two magnon scattering process.

There are a few important points to be made. Equation (2) seems to have a wide applicability in metallic ferromagnetic films. The presence of a zero frequency offset, $\Delta H(0)$, in the FMR linewidth can be significant and will generally result in a considerably higher effective damping than expected from the intrinsic contribution. This is particularly important in magnetization reversal studies where the effective frequency range is below $10 \mathrm{GHz}$. The relaxation times used in micro- magnetic modelling of dynamic magnetization reversal processes should be compared to FMR measurements only when the FMR measurements are taken over a wide range of microwave frequencies allowing one to estimate effective damping below $10 \mathrm{GHz}$.

\section{ACKNOWLEDGMENTS}

Financial support from the Natural Sciences and Engineering Research Council of Canada (NSERC) and the Canadian Institute for Advanced Research (CIAR) is gratefully acknowledged. B.H. also expresses thanks to the Alexander Humboldt Foundation for support during his stay at Max Planck Institute in Halle.
${ }^{1}$ B. Heinrich and J. F. Cochran, Adv. Phys. 42, 523 (1993).

${ }^{2}$ B. Heinrich, D. J. Meredith, and J. F. Cochran, J. Appl. Phys. 50, 7726 (1979).

${ }^{3}$ M. Sparks, Ferromagnetic Relaxation Theory (McGraw-Hill, New York, 1966).

${ }^{4}$ E. Schlömann, Phys. Chem. Solids 6, 242 (1958).

${ }^{5}$ R. LeCraw, E. G. Spencer, and C. S. Porter, Phys. Rev. 110, 1311 (1958).

${ }^{6}$ S. Geschwind and A. M. Clogston, Phys. Rev. 108, 49 (1957).

${ }^{7}$ M. J. Hurben, D. R. Franklin, and C. E. Patton, J. Appl. Phys. 81, 7458 (1997).

${ }^{8}$ C. E. Patton, C. H. Wilts, and F. B. Humphrey, J. Appl. Phys. 38, 1358 (1967).

${ }^{9}$ B. Heinrich, J. M. Rudd, K. Urquhart, K. Myrtle, and J. F. Cochran, J. Appl. Phys. 55, 1814 (1984).

${ }^{10}$ C. E. Patton and C. H. Wilts, J. Appl. Phys. 38, 3537 (1967).

${ }^{11}$ B. Heinrich, K. B. Urquhart, A. S. Arrott, J. F. Cochran, K. Myrtle, and S. T. Purcell, Phys. Rev. Lett. 59, 1756 (1987).
${ }^{12}$ F. Schreiber, J. Pflaum, Z. Frait, T. Mühge, and J. Pelzl, Solid State Commun. 93, 965 (1995).

${ }^{13}$ B. Heinrich, J. F. Cochran, and R. Hasegawa, J. Appl. Phys. 57, 3690 (1985).

${ }^{14}$ R. D. McMichael, M. D. Stiles, P. J. Chen, and W. F. Egelhoff, Jr., J. Appl. Phys. 83, 7037 (1998).

${ }^{15}$ R. Arias and D. L. Mills, Phys. Rev. B 60, 7395 (1999).

${ }^{16}$ A. Azevedo, A. B. Oliveira, F. M. de Aguiar, and S. M. Rezende, Phys. Rev. B 62, 5331 (2000).

${ }^{17}$ T. L. Monchesky, B. Heinrich, R. Urban, K. Myrtle, M. Klaua, and J. Kirschner, Phys. Rev. B 60, 10242 (1999).

${ }^{18}$ T. Monchesky, R. Urban, B. Heinrich, M. Klaua, and J. Kirschner, J. Appl. Phys. 87, 5167 (2000).

${ }^{19}$ M. J. Hurben and C. E. Patton, J. Appl. Phys. 83, 4344 (1998).

${ }^{20}$ The perpendicular and in-plane configuration corresponds to the magnetic moment oriented perpendicular and parallel to the film surface, respectively. 